\title{
TIGHT BOUNDS ON EXPECTED ORDER STATISTICS
}

\author{
DiMITRIS BERTSIMAS \\ Massachusetts Institute of Technology \\ Cambridge, MA 02139 \\ E-mail:dbertsim@mit.edu \\ KARTHIK NATARAJAN \\ Department of Mathematics \\ National University of Singapore \\ Singapore 117543 \\ E-mail: matkbn@nus.edu.sg \\ Chung-Piaw Teo \\ Department of Decision Sciences \\ NUS Business School \\ Singapore 117591 \\ E-mail: bizteocp@nus.edu.sg
}

Sloan School of Management and Operations Research Center

In this article, we study the problem of finding tight bounds on the expected value of the $k$ th-order statistic $E\left[X_{k: n}\right]$ under first and second moment information on $n$ real-valued random variables. Given means $E\left[X_{i}\right]=\mu_{i}$ and variances $\operatorname{Var}\left[X_{i}\right]=\sigma_{i}^{2}$, we show that the tight upper bound on the expected value of the highest-order statistic $E\left[X_{n: n}\right]$ can be computed with a bisection search algorithm. An extremal discrete distribution is identified that attains the bound, and two closed-form bounds are proposed. Under additional covariance information $\operatorname{Cov}\left[X_{i}, X_{j}\right]=Q_{i j}$, we show that the tight upper bound on the expected value of the highest-order statistic can be computed with semidefinite optimization. We generalize these results to find bounds on the expected value of the $k$ th-order statistic under mean and variance information. For $k<n$, this bound is shown to be tight under identical means and variances. All of our results are distributionfree with no explicit assumption of independence made. Particularly, using optimization methods, we develop tractable approaches to compute bounds on the expected value of order statistics. 


\section{INTRODUCTION}

Let $X=\left(X_{1}, \ldots, X_{n}\right)$ denote $n \geq 2$ jointly distributed real-valued random variables. The order statistics of this set is a reordering of the $X_{i}$ in terms of nondecreasing values, expressed as $X_{1: n} \leq \cdots \leq X_{k: n} \leq \cdots \leq X_{n: n}$. The lowest- and highest-order statistics are denoted by $X_{1: n}$ and $X_{n: n}$, respectively. One of the central problems in statistics is to find, bound, or approximate the expected value of order statistics under varying assumptions on the distribution of the random variables. For detailed reviews on this subject, the reader is referred to [9] and [2].

In this article, we focus on finding bounds on the expected value of order statistics under moment information on the random variables. Let $\boldsymbol{X} \sim{ }_{\theta} \boldsymbol{m}$ denote the set of feasible distributions $\theta$ that satisfies the given moments $\boldsymbol{m}$ for the random variables.

DEFINITION 1: $Z_{k: n}^{*}$ is a tight upper bound on the expected value of the kth-order statistic if

$$
Z_{k: n}^{*}=\sup _{X \sim{ }_{\theta} m} E_{\theta}\left[X_{k: n}\right]
$$

that is, there exists a feasible distribution or a limit of a sequence of feasible distributions that achieves the upper bound.

No other assumptions on independence or the type of distribution are made. In this article, we develop methods to compute $Z_{k: n}^{*}$ under first and second moment information on the random variables. Next, we review some of the classical bounds for order statistics.

\subsection{Some Known Bounds}

Given identical means and variances $\left(\mu, \sigma^{2}\right)$ for the random variables, one of the earliest known bounds for the expected highest-order statistic was derived by Gumbel [10] and Hartley and David [11]. Under the assumption of independence, they obtained the upper bound $\mu+\sigma(n-1) / \sqrt{2 n-1}$. Moriguti [18] extended this result to the special case of symmetrically distributed random variables.

For more general distributions (not necessarily independent or identically distributed), Arnold and Groeneveld [3] obtained an upper bound on the expected value of the $k$ th-order statistic:

$$
E_{\theta}\left[X_{k: n}\right] \leq \frac{\sum_{i=1}^{n} \mu_{i}}{n}+\sqrt{\frac{k-1}{n(n-k+1)} \sum_{i=1}^{n}\left[\sigma_{i}^{2}+\left(\mu_{i}-\frac{\sum_{i=1}^{n} \mu_{i}}{n}\right)^{2}\right]} .
$$

Under identical means and variances, this bound reduces to

$$
E_{\theta}\left[X_{k: n}\right] \leq \mu+\sigma \sqrt{\frac{k-1}{n-k+1}} .
$$


For this particular case, Arnold and Groeneveld [3] showed that (2) is tight by explicitly constructing a distribution that achieved the bound. However, for general meanvariance information, (1) is not necessarily tight. Aven [4] proposed an alternative upper bound on the expected value of the highest-order statistic:

$$
E_{\theta}\left[X_{n: n}\right] \leq \max _{1 \leq i \leq n} \mu_{i}+\sqrt{\frac{n-1}{n} \sum_{i=1}^{n} \sigma_{i}^{2}} .
$$

This bound is also not tight under general mean-variance information. In this article, we develop an algorithmic approach to find (possibly) tight bounds on the expected value of the order statistic $Z_{k: n}^{*}$. We characterize cases for which the bound can be computed tractably, or we propose simple closed-form bounds that seem promising.

\subsection{Contributions}

Our main contributions in this article are as follows:

1. In Section 2 we find the tight upper bound on the expected value of the highest-order statistic $Z_{n: n}^{*}$ under mean-variance information on the random variables. An efficiently solvable bisection search approach is developed to compute $Z_{n: n}^{*}$. A discrete extremal distribution is identified that attains the tight bound. Two simple closed-form bounds for the expected highest-order statistic are proposed. Under additional covariance information, we propose a semidefinite programming approach to find the tight bound on the expected highest-order statistic.

2. In Section 3 we extend the bisection search method to obtain bounds on the expected value of the general $k$ th-order statistic under mean-variance information. For $k<n$, we show that the bound is tight under identical means and variances. For general mean-variance information, the bound found with the bisection search method, although not necessarily tight, is at least as strong as (1).

3. In Section 4 we provide computational experiments to test the performance of the different bounds.

\section{BOUNDS ON EXPECTED HIGHEST-ORDER STATISTIC}

We first compute the tight upper bound on the expected highest-order statistic $Z_{n: n}^{*}$ under mean-variance information on the random variables. The mean and variance information on the random variables are denoted as $\boldsymbol{\mu}=\left(\mu_{1}, \ldots, \mu_{n}\right)$ and $\boldsymbol{\sigma}^{\mathbf{2}}=$ $\left(\sigma_{1}^{2}, \ldots, \sigma_{n}^{2}\right)$. The set of feasible distributions satisfying these moment restrictions is represented by $\boldsymbol{X} \sim_{\theta}\left(\boldsymbol{\mu}, \boldsymbol{\sigma}^{2}\right)$. For simplicity of presentation, we will assume that all of the $\sigma_{i}$ are strictly positive. As discussed later, this condition can in fact be relaxed. 
The approach to compute the tight upper bound on the expected value of the highest-order statistic is based on a convex reformulation technique, initially proposed by Meilijson and Nadas [17] and developed later in Bertsimas, Natarajan, and Teo [5]. The reformulation is based on the observation that the highest-order statistic $X_{n: n}$ is a convex function in the $X_{i}$ variables. We review the key ideas of this reformulation next.

THEOREM 1 (Bertsimas et al. [5]): The tight upper bound on the expected value of the highest-order statistic $Z_{n: n}^{*}$ given $\boldsymbol{X} \sim_{\theta}\left(\boldsymbol{\mu}, \boldsymbol{\sigma}^{2}\right)$ is obtained by solving

$$
Z_{n: n}^{*}=\min _{z}\left(z_{n: n}+\sum_{i=1}^{n} \sup _{X_{i} \sim \theta_{i}\left(\mu_{i}, \sigma_{i}^{2}\right)} E_{\theta_{i}}\left[X_{i}-z_{i}\right]^{+}\right),
$$

where $x^{+}=\max (0, x)$.

Sketch of Proof: We first show that (4) provides an upper bound on $Z_{n: n}^{*}$. To see this, note that we have the following inequality for each variable $X_{i}$ :

$$
\begin{aligned}
X_{i} & =z_{i}+\left(X_{i}-z_{i}\right) \\
& \leq z_{n: n}+\sum_{i=1}^{n}\left[X_{i}-z_{i}\right]^{+} .
\end{aligned}
$$

Since the right-hand side of this inequality is independent of the particular $i$, we have

$$
X_{n: n} \leq z_{n: n}+\sum_{i=1}^{n}\left[X_{i}-z_{i}\right]^{+}
$$

Taking expectations and minimizing over the $z_{i}$ variables, we obtain the best upper bound:

$$
E_{\theta}\left[X_{n: n}\right] \leq \min _{z}\left(z_{n: n}+\sum_{i=1}^{n} E_{\theta}\left[X_{i}-z_{i}\right]^{+}\right) .
$$

Optimizing over distributions with given mean-variance information, we obtain an upper bound:

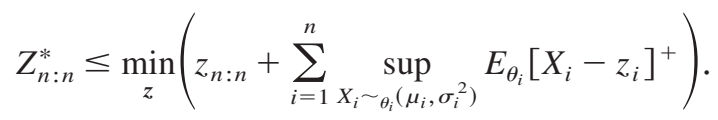

Note that the inner problem is optimization over probability distributions of single random variables $\theta_{i}$, since no cross-moment information is specified. For a proof that the bound is tight, the reader is referred to [5]. Alternatively, we construct an extremal distribution in Theorem 3 that attains the bound. 
The solution for the inner problem in (4) is in fact known in closed form from [13] and [22]. We now outline a simple proof for this bound.

Proposition 1: The tight upper bound on the expected value $E_{\theta_{i}}\left[X_{i}-z_{i}\right]^{+}$given $X_{i} \sim_{\theta_{i}}\left(\mu_{i}, \sigma_{i}^{2}\right)$ is

$$
\sup _{X_{i} \sim \theta_{i}\left(\mu_{i}, \sigma_{i}^{2}\right)} E_{\theta_{i}}\left[X_{i}-z_{i}\right]^{+}=\frac{1}{2}\left[\mu_{i}-z_{i}+\sqrt{\left(\mu_{i}-z_{i}\right)^{2}+\sigma_{i}^{2}}\right] .
$$

Proof: We have the basic equality

$$
\left[X_{i}-z_{i}\right]^{+}=\frac{1}{2}\left(X_{i}-z_{i}+\left|X_{i}-z_{i}\right|\right)
$$

Taking expectations, we obtain

$$
\begin{aligned}
E_{\theta_{i}}\left[X_{i}-z_{i}\right]^{+} & =\frac{1}{2}\left(E_{\theta_{i}}\left[X_{i}-z_{i}\right]+E_{\theta_{i}}\left|X_{i}-z_{i}\right|\right), \quad \forall X_{i} \sim_{\theta_{i}}\left(\mu_{i}, \sigma_{i}^{2}\right) \\
& \leq \frac{1}{2}\left(\mu_{i}-z_{i}+\sqrt{\left(\mu_{i}-z_{i}\right)^{2}+\sigma_{i}^{2}}\right),
\end{aligned}
$$

(from the Cauchy-Schwarz inequality).

Furthermore, this bound can be shown to be tight since it is attained by the distribution

$$
X_{i}= \begin{cases}z_{i}+\sqrt{\left(\mu_{i}-z_{i}\right)^{2}+\sigma_{i}^{2}}, \quad \text { w.p. } p=\frac{1}{2}\left(1+\frac{\mu_{i}-z_{i}}{\sqrt{\left(\mu_{i}-z_{i}\right)^{2}+\sigma_{i}^{2}}}\right) \\ z_{i}-\sqrt{\left(\mu_{i}-z_{i}\right)^{2}+\sigma_{i}^{2}}, & \text { w.p. } 1-p=\frac{1}{2}\left(1-\frac{\mu_{i}-z_{i}}{\sqrt{\left(\mu_{i}-z_{i}\right)^{2}+\sigma_{i}^{2}}}\right) .\end{cases}
$$

Using this closed-form bound, we now show that the tight upper bound on the expected highest-order statistic can be found by solving a univariate convex minimization problem.

THEOREM 2: The tight upper bound on the expected value of the highest-order statistic $Z_{n: n}^{*}$ given $\boldsymbol{X} \sim_{\theta}\left(\boldsymbol{\mu}, \boldsymbol{\sigma}^{2}\right)$ is obtained by solving the strictly convex univariate minimization problem

$$
Z_{n: n}^{*}=\min _{z} f_{n: n}(z)=\min _{z}\left(z+\sum_{i=1}^{n} \frac{1}{2}\left[\mu_{i}-z+\sqrt{\left(\mu_{i}-z\right)^{2}+\sigma_{i}^{2}}\right]\right)
$$


Proof: Combining Theorem 1 and Proposition 1, the tight upper bound on the expected highest-order statistic is

$$
Z_{n: n}^{*}=\min _{z}\left(z_{n: n}+\sum_{i=1}^{n} \frac{1}{2}\left[\mu_{i}-z_{i}+\sqrt{\left(\mu_{i}-z_{i}\right)^{2}+\sigma_{i}^{2}}\right]\right) .
$$

We next show that (7) can be simplified to a single-variable optimization problem. Let $z^{*}$ be an optimal solution to (7) and let $z_{n: n}^{*}$ denote the highest-order statistic. Note that the second term, $\sum_{i=1}^{n} \frac{1}{2}\left[\mu_{i}-z_{i}+\sqrt{\left(\mu_{i}-z_{i}\right)^{2}+\sigma_{i}^{2}}\right]$, is decreasing in $z_{i}$. Hence, for any $i<n$ with $z_{i: n}^{*}<z_{n: n}^{*}$, by increasing $z_{i: n}^{*}$ up to $z_{n: n}^{*}$ the first term remains unaffected while the second term decreases, thus reducing the objective. Since we are minimizing the objective, the optimal solution will set all of the $z_{i}^{*}$ values equal to $z_{n: n}^{*}$.

It can be easily checked that $f_{n: n}$ is a strictly convex function, implying that the function has a unique global minimum. The optimal decision variable $z$ in (6) hence satisfies the first-order condition obtained by setting the derivative $\partial f_{n: n}(z) / \partial z$ to zero:

$$
\frac{\partial f_{n: n}\left(z^{*}\right)}{\partial z}=\sum_{i=1}^{n}\left(\frac{z^{*}-\mu_{i}}{\sqrt{\left(\mu_{i}-z^{*}\right)^{2}+\sigma_{i}^{2}}}\right)-(n-2)=0 .
$$

Remark: Our result can be viewed as an extension of the bound from Lai and Robbins [15] and Ross [21]. In their case, under completely known marginal distributions $X_{i} \sim{ }_{\theta} \theta_{i}$, they obtain the following tight bound on the highest-order statistic:

$$
\sup _{X_{i} \sim \theta_{\theta}, \forall i} E_{\theta}\left[X_{n: n}\right]=\min _{z}\left(z+\sum_{i=1}^{n} E_{\theta_{i}}\left[X_{i}-z\right]^{+}\right) .
$$

Note that this result follows also from Meilijson and Nadas [17].

\subsection{An Extremal Probability Distribution}

We construct an $n$-atom discrete distribution that satisfies that mean-variance requirements and attains the bound in (6).

TheORem 3: Let $z^{*}$ denote the optimal minimizer to (6). An $n$-atom extremal distribution for $\boldsymbol{X}$ that achieves the upper bound and satisfies the mean-variance requirements is

$$
\boldsymbol{X}=\left\{\boldsymbol{X}^{(j)}, \quad \text { w.p. } p_{j}=\frac{1}{2}\left(1+\frac{\mu_{j}-z^{*}}{\sqrt{\left(\mu_{j}-z^{*}\right)^{2}+\sigma_{j}^{2}}}\right) \quad \text { for } j=1, \ldots, n,\right.
$$


where $\boldsymbol{X}^{(j)}=\left(X_{1}^{(j)}, \ldots, X_{n}^{(j)}\right)$ is expressed as

$$
X_{i}^{(j)}= \begin{cases}z^{*}+\sqrt{\left(\mu_{i}-z^{*}\right)^{2}+\sigma_{i}^{2}} & \text { if } i=j \\ z^{*}-\sqrt{\left(\mu_{i}-z^{*}\right)^{2}+\sigma_{i}^{2}} & \text { if } i \neq j .\end{cases}
$$

Proof: From the definition, it is clear that the $p_{j}$ values denote a probability measure since $p_{j} \geq 0$ for all $j$ and

$$
\begin{aligned}
\sum_{j=1}^{n} p_{j} & =\sum_{j=1}^{n} \frac{1}{2}\left(1+\frac{\mu_{j}-z^{*}}{\sqrt{\left(\mu_{j}-z^{*}\right)^{2}+\sigma_{i}^{2}}}\right) \\
& =\frac{n}{2}+\frac{2-n}{2} \quad(\text { from }(8)) \\
& =1 .
\end{aligned}
$$

It can be verified for this $n$-atom distribution that

$$
\begin{gathered}
E_{\theta}\left[X_{i}\right]=\sum_{j=1}^{n} p_{j} X_{i}^{(j)}=\mu_{i}, \quad i=1, \ldots, n, \\
\operatorname{Var}_{\theta}\left[X_{i}\right]=\sum_{j=1}^{n} p_{j}\left(X_{i}^{(j)}-\mu_{i}\right)^{2}=\sigma_{i}^{2}, \quad i=1, \ldots, n .
\end{gathered}
$$

Furthermore, the maximum among the $n$ random variables for the $j$ th atom is attained by $X_{j}^{(j)}$. Thus,

$$
E_{\theta}\left[X_{n: n}\right]=\sum_{j=1}^{n} p_{j} X_{j}^{(j)}=\left(z^{*}+\sum_{j=1}^{n} \frac{1}{2}\left[\mu_{j}-z^{*}+\sqrt{\left(\mu_{j}-z^{*}\right)^{2}+\sigma_{j}^{2}}\right]\right)=f_{n: n}\left(z^{*}\right) .
$$

This $n$-atom distribution attains the upper bound on the expected value of the highestorder statistic and satisfies the mean and variance requirements. This verifies that the bound in Theorem 1 is tight.

\subsection{Solution Techniques}

In general, it does not seem possible to find $Z_{n: n}^{*}$ in closed form. A special case under which this is possible is discussed next.

2.2.1. Identical Mean and Variance For identical mean-variance pairs $\left(\mu, \sigma^{2}\right)$, solving (8) yields the optimal value for $z^{*}$ :

$$
z^{*}=\mu+\sigma \frac{n-2}{2 \sqrt{n-1}}
$$


Substituting this into (6) yields the tight bound

$$
\sup _{X_{i} \sim{ }_{\theta}\left(\mu, \sigma^{2}\right) \forall i} E_{\theta}\left[X_{n: n}\right]=\mu+\sigma \sqrt{n-1}
$$

Note that this is exactly (2) obtained by Arnold and Groeneveld [3] for $k=n$. A distribution that attains this bound is randomly selecting $n$ elements without replacement from the set in which one element has the value $\mu+\sigma \sqrt{n-1}$ and the remaining $n-1$ elements have the value $\mu-\sigma / \sqrt{n-1}$.

2.2.2. General Mean-Variance Pairs For the general case, we outline a simple bisection search algorithm to find $Z_{n: n}^{*}$.

Description of the Algorithm

1. Initialize $z_{l}$ and $z_{u}$ such that $\partial f_{n: n}\left(z_{l}\right) / \partial z \leq 0$ and $\partial f_{n: n}\left(z_{u}\right) / \partial z \geq 0$ and $\epsilon>0$ to a given tolerance level.

2. Let $z=\left(z_{l}+z_{u}\right) / 2$.

3. While $\left|\partial f_{n: n}(z) / \partial z\right| \geq \epsilon$, do:

(a) If $\partial f_{n: n}(z) / \partial z \geq 0$, set $z_{u}=z$; or else set $z_{l}=z$.

(b) Go back to Step 2 .

4. Output $Z_{n: n}^{*}=f_{n: n}(z)$.

We propose two simple upper and lower bounds $z_{u}$ and $z_{l}$ on the range of the optimal $z^{*}$ to initialize the algorithm. Consider the problem of finding a $z_{u}$ such that $f^{\prime}\left(z_{u}\right) \geq 0$. One such $z_{u}$ is constructed such that each term on the left-hand side of (8) contributes at least a fraction $(n-2) / n$ :

$$
\frac{z_{u}-\mu_{i}}{\sqrt{\left(\mu_{i}-z_{u}\right)^{2}+\sigma_{i}^{2}}} \geq \frac{n-2}{n}, \quad i=1, \ldots, n
$$

which reduces to

$$
z_{u} \geq \mu_{i}+\sigma_{i} \frac{n-2}{2 \sqrt{n-1}}, \quad i=1, \ldots, n
$$

We choose $z_{u}$ as

$$
z_{u}=\max _{1 \leq i \leq n}\left(\mu_{i}+\sigma_{i} \frac{n-2}{2 \sqrt{n-1}}\right) .
$$

Similarly, a lower bound $z_{l}$ can be found such that

$$
\frac{z_{l}-\mu_{i}}{\sqrt{\left(\mu_{i}-z_{l}\right)^{2}+\sigma_{i}^{2}}} \leq \frac{n-2}{n}, \quad i=1, \ldots, n
$$


A $z_{l}$ that satisfies this condition is

$$
z_{l}=\min _{1 \leq i \leq n}\left(\mu_{i}+\sigma_{i} \frac{n-2}{2 \sqrt{n-1}}\right) .
$$

Our computational tests indicate that these values of $z_{u}$ and $z_{l}$ lead quickly to the tight bound.

2.2.3. New Closed-Form Bounds Based on the two end points, we now propose simple closed-form bounds on the expected value of the highest-order statistic.

THEOREM 4: Two closed-form upper bounds on the expected value of the highestorder statistic given $\boldsymbol{X} \sim_{\theta}\left(\boldsymbol{\mu}, \boldsymbol{\sigma}^{2}\right)$ are

$$
\begin{aligned}
& \frac{1}{2}\left(\sum_{i=1}^{n}\left[\mu_{i}+\sqrt{\left(\mu_{i}-\max _{1 \leq i \leq n}\left\{\mu_{i}+\frac{n-2}{2 \sqrt{n-1}} \sigma_{i}\right\}\right)^{2}+\sigma_{i}^{2}}\right]\right. \\
& \left.+(2-n)\left[\max _{1 \leq i \leq n}\left\{\mu_{i}+\frac{n-2}{2 \sqrt{n-1}} \sigma_{i}\right\}\right]\right), \\
& \frac{1}{2}\left(\sum_{i=1}^{n}\left[\mu_{i}+\sqrt{\left(\mu_{i}-\min _{1 \leq i \leq n}\left\{\mu_{i}+\frac{n-2}{2 \sqrt{n-1}} \sigma_{i}\right\}\right)^{2}+\sigma_{i}^{2}}\right]\right. \\
& \left.+(2-n)\left[\min _{1 \leq i \leq n}\left\{\mu_{i}+\frac{n-2}{2 \sqrt{n-1}} \sigma_{i}\right\}\right]\right) .
\end{aligned}
$$

Proof: Substitute $z=z_{l}$ and $z=z_{u}$ in (6) respectively.

Note that (13) and (14) reduce to the tight upper bound (10) on the expected highest-order statistic for random variables with identical mean-variance pairs.

\subsection{Extensions}

We now extend the results to the case where some of the $\sigma_{i}^{2}=0$ (i.e., $X_{i}$ is deterministic). Without loss of generality, we assume that exactly one variable is deterministic since the case with multiple constants can be reduced to this case by choosing the maximum of the constants. Given $n \geq 1$ random variables with strictly positive variances and a constant $K$, we want to find the tight upper bound on $E_{\theta}\left[\max \left(X_{n: n}, K\right)\right]$. By introducing an extra decision variable, $z_{n+1}$, for the term $K$, (4) reduces to

$$
\begin{aligned}
& \sup _{\boldsymbol{X} \sim \sim_{\theta}\left(\boldsymbol{\mu}, \boldsymbol{\sigma}^{2}\right)} E_{\theta}\left[\max \left(X_{n: n}, K\right)\right] \\
& \quad=\min _{z}\left(z_{n+1: n+1}+\sum_{i=1}^{n} \frac{1}{2}\left[\mu_{i}-z_{i}+\sqrt{\left(\mu_{i}-z_{i}\right)^{2}+\sigma_{i}^{2}}\right]+\left(K-z_{n+1}\right)^{+}\right) .
\end{aligned}
$$


Using an argument similar to Theorem 2, it can be checked that the optimal solution will set all of the $z_{i}$ values the same at a value greater than or equal to $K$. Hence, the tight upper bound on the expected highest-order statistic is

$$
\sup _{\boldsymbol{X} \sim_{\theta}\left(\boldsymbol{\mu}, \boldsymbol{\sigma}^{2}\right)} E_{\theta}\left[\max \left(X_{n: n}, K\right)\right]=\min _{z \geq K}\left(z+\sum_{i=1}^{n} \frac{1}{2}\left[\mu_{i}-z+\sqrt{\left(\mu_{i}-z\right)^{2}+\sigma_{i}^{2}}\right]\right),
$$

which reduces to the constrained version of (6):

$$
\sup _{\boldsymbol{X} \sim \sim_{\theta}\left(\boldsymbol{\mu}, \boldsymbol{\sigma}^{2}\right)} E_{\theta}\left[\max \left(X_{n: n}, K\right)\right]=\min _{z \geq K} f_{n: n}(z) .
$$

The tight upper bound can be found by a modified bisection search method:

1. Solve the unconstrained version of (16) with bisection search to find $z^{*}$.

2. Output $f_{n: n}\left(\max \left(z^{*}, K\right)\right)$.

We propose using the following two closed-form bounds in this case:

$$
f_{n: n}\left[\max \left(\max _{1 \leq i \leq n}\left\{\mu_{i}+\frac{n-2}{2 \sqrt{n-1}} \sigma_{i}\right\}, K\right)\right]
$$

and

$$
f_{n: n}\left[\max \left(\min _{1 \leq i \leq n}\left\{\mu_{i}+\frac{n-2}{2 \sqrt{n-1}} \sigma_{i}\right\}, K\right)\right]
$$

\subsection{Extensions to Additional Covariance Information}

In this subsection, we propose an algorithmic approach to find the tight upper bound on the expected value of the highest-order statistic under covariance information. Given the mean and covariance matrix for the random variables $\boldsymbol{X} \sim_{\theta}(\boldsymbol{\mu}, \boldsymbol{Q})$, the tight upper bound is computed by finding a distribution $\theta$ that solves

$$
\begin{aligned}
Z_{n: n}^{*}= & \sup _{\theta} E_{\theta}\left[X_{n: n}\right] \\
& \text { s.t. } E_{\theta}[\boldsymbol{X}]=\boldsymbol{\mu} \\
& E_{\theta}\left[\boldsymbol{X} \boldsymbol{X}^{\prime}\right]=\boldsymbol{Q}+\boldsymbol{\mu} \boldsymbol{\mu}^{\prime}, \\
& E_{\theta}\left[\mathbb{I}_{\mathfrak{R}^{n}}\right]=1 .
\end{aligned}
$$

Here $\mathbb{I}_{\Re^{n}}(\boldsymbol{X})=1$ if $\boldsymbol{X} \in \Re^{n}$ and zero otherwise represents the indicator function. This problem has been well studied under the class of moment problems in Isii [12] and Karlin and Studden [14]. To solve (19), we construct the dual problem by introducing variables $\boldsymbol{y}, \boldsymbol{Y}$, and $y_{0}$ for each of the moment constraints. The dual problem is formulated as 


$$
\begin{aligned}
Z^{*}= & \min \left(\boldsymbol{y}^{\prime} \boldsymbol{\mu}+\boldsymbol{Y} \cdot\left(\boldsymbol{Q}+\boldsymbol{\mu} \boldsymbol{\mu}^{\prime}\right)+y_{0}\right) \\
& \text { s.t. } \boldsymbol{y}^{\prime} \boldsymbol{X}+\boldsymbol{X}^{\prime} \boldsymbol{Y} \boldsymbol{X}+y_{0} \geq X_{n: n}, \quad \forall \boldsymbol{X} \in \mathfrak{R}^{n} .
\end{aligned}
$$

The constraints in (20) imply the nonnegativity of a quadratic function over $\Re^{n}$. By taking the expectation of the dual constraints, it is easy to see that $Z^{*} \geq Z_{n: n}^{*}$. Furthermore, Isii [12] showed that if the covariance matrix $\boldsymbol{Q}>\mathbf{0}$ is strictly positive definite, then $Z^{*}=Z_{n: n}^{*}$. Under this assumption, the convexity of $X_{n: n}$ implies that the tight upper bound on the expected highest-order statistic is

$$
\begin{aligned}
Z_{n: n}^{*}= & \min \left(\boldsymbol{y}^{\prime} \boldsymbol{\mu}+\boldsymbol{Y} \cdot\left(\boldsymbol{Q}+\boldsymbol{\mu} \boldsymbol{\mu}^{\prime}\right)+y_{0}\right) \\
& \text { s.t. } \boldsymbol{y}^{\prime} \boldsymbol{X}+\boldsymbol{X}^{\prime} \boldsymbol{Y} \boldsymbol{X}+y_{0} \geq X_{i}, \quad i=1, \ldots, n, \forall \boldsymbol{X} \in \Re^{n} .
\end{aligned}
$$

Let $e^{(i)}$ denote a unit vector with the $i$ th component $e_{i}^{(i)}=1$ and zero otherwise. The equivalence between the global nonnegativity of a quadratic polynomial and the semidefinite representation [20] implies that (21) can be rewritten as

$$
\begin{aligned}
Z_{n: n}^{*}= & \min \left(\boldsymbol{y}^{\prime} \boldsymbol{\mu}+\boldsymbol{Y} \cdot\left(\boldsymbol{Q}+\boldsymbol{\mu} \boldsymbol{\mu}^{\prime}\right)+y_{0}\right) \\
& \text { s.t. }\left(\begin{array}{cc}
\boldsymbol{Y} & \left(\boldsymbol{y}-\boldsymbol{e}_{\boldsymbol{i}}\right) / 2 \\
\left(\boldsymbol{y}-\boldsymbol{e}_{\boldsymbol{i}}\right)^{\prime} / 2 & y_{0}
\end{array}\right) \geqslant 0, \quad i=1, \ldots, n .
\end{aligned}
$$

Here $A \geqslant 0$ denotes the constraint that the matrix $A$ is positive semidefinite. Formulation (22) is a semidefinite optimization problem that can be solved within $\epsilon>0$ of the optimal solution in polynomial time in the problem data and $\log (1 / \epsilon)$ [19]. In practice, standard semidefinite optimization codes such as SeDuMi [23] can be used to find the tight upper bound on the expected highest-order statistic under covariance information.

\section{BOUNDS ON EXPECTED $k$ TH-ORDER STATISTIC}

In this section, we generalize our results to find bounds on the expected value of the $k$ th-order statistic for $k<n$ under mean-variance information on the random variables; that is,

$$
Z_{k: n}^{*}=\sup _{\boldsymbol{X} \sim \sim_{\theta}\left(\boldsymbol{\mu}, \boldsymbol{\sigma}^{2}\right)} E_{\theta}\left[X_{k: n}\right]
$$

Our results are based on the simple observation that

$$
X_{k: n} \leq \frac{\sum_{i=k}^{n} X_{i: n}}{n-k+1} .
$$

We find tight bounds on the expected value of the right-hand side of (23) to obtain bounds on the expected value of the $k$ th-order statistic. 
THEOREM 5: The tight upper bound on the expected value of the sum of the kth-to nth-order statistic given $\boldsymbol{X} \sim_{\theta}\left(\boldsymbol{\mu}, \boldsymbol{\sigma}^{2}\right)$ is obtained by solving

$$
\sup _{\boldsymbol{X} \sim{ }_{\theta}\left(\boldsymbol{\mu}, \boldsymbol{\sigma}^{2}\right)} E_{\theta}\left[\sum_{i=k}^{n} X_{i: n}\right]=\min _{z}\left((n-k+1) z+\sum_{i=1}^{n} \frac{1}{2}\left[\mu_{i}-z+\sqrt{\left(\mu_{i}-z\right)^{2}+\sigma_{i}^{2}}\right]\right) .
$$

Proof: Using the result from Bertsimas et al. [5], the upper bound on the sum of the expected value of the $k$ th- to $n$ th-order statistic is

$$
\sup _{\boldsymbol{X} \sim{ }_{\theta}\left(\boldsymbol{\mu}, \boldsymbol{\sigma}^{2}\right)} E_{\theta}\left[\sum_{i=k}^{n} X_{i: n}\right]=\min _{z}\left(\sum_{i=k}^{n} z_{i: n}+\sum_{i=1}^{n} \frac{1}{2}\left[\mu_{i}-z_{i}+\sqrt{\left(\mu_{i}-z_{i}\right)^{2}+\sigma_{i}^{2}}\right]\right) .
$$

As earlier, (25) can be reduced to a single-variable optimization problem. To see this, let $z^{*}$ be an optimal solution to (25). For any $l<k$ with $z_{l: n}^{*}<z_{k: n}^{*}$, we can increase $z_{l: n}^{*}$ to $z_{k: n}^{*}$ since the first term is unaffected $\left(\sum_{i=k}^{n} z_{i: n}^{*}\right.$ is unaffected by change in $z_{l: n}^{*}$, for $l<k$, provided $z_{l: n}^{*}<z_{k: n}^{*}$ ) while the second term decreases in $z_{l: n}^{*}$. Hence, we have $z_{l: n}^{*}=z_{k: n}^{*}$ for $l<k$. Furthermore, for $l>k$ with $z_{l: n}^{*}>z_{k: n}^{*}$, by decreasing $z_{l: n}^{*}$ to $z_{k: n}^{*}$ the first term decreases at a rate of 1 while the second term increases at a rate of at most 1 . Since we want to minimize our objective, we have $z_{l: n}^{*}=z_{k: n}^{*}$ for $l=1, \ldots, n$.

Using (23) and Theorem 5, we now obtain a bound on the expected $k$ th-order statistic.

THEOREM 6: An upper bound on the expected value of the kth-order statistic $Z_{k: n}^{*}$ given $\boldsymbol{X} \sim_{\theta}\left(\boldsymbol{\mu}, \boldsymbol{\sigma}^{\mathbf{2}}\right)$ is obtained by solving

$$
Z_{k: n}^{*} \leq \min _{z} f_{k: n}(z)=\min _{z}\left(z+\frac{1}{2(n-k+1)} \sum_{i=1}^{n}\left[\mu_{i}-z+\sqrt{\left(\mu_{i}-z\right)^{2}+\sigma_{i}^{2}}\right]\right) .
$$

Note that the nonconvex structure of the $k$ th-order statistic for $k<n$ implies that (26) is not necessarily tight for general mean-variance pairs. However, (26) is at least as tight as (1) proposed by Arnold and Groeneveld [3]. This follows from observing that they obtained their bound by bounding (23), although not in the tightest manner. A special case under which (26) is tight is described next.

3.1. Identical Mean and Variance For identical mean-variance pairs $\left(\mu, \sigma^{2}\right)$, (26) yields the optimal value for $z^{*}$ :

$$
z^{*}=\mu+\sigma \frac{2 k-n-2}{2 \sqrt{(k-1)(n-k+1)}} .
$$


Substituting this into (26) yields

$$
\sup _{X_{i} \sim \theta} E_{\theta}\left[\sigma_{\left.\sigma^{2}\right), \forall i}\left[X_{k: n}\right] \leq \mu+\sigma \sqrt{\frac{k-1}{n-k+1}} .\right.
$$

This is exactly (2) obtained by Arnold and Groeneveld [3]. To see that (27) is tight, consider a distribution obtained by randomly selecting $n$ elements without replacement from the set in which $n-k+1$ elements have the value $\mu+$ $\sigma \sqrt{(k-1) /(n-k+1)}$ and the remaining $k-1$ elements have the value $\mu-$ $\sigma \sqrt{(n-k+1) /(k-1)}$. It is easy to verify that this distribution attains the bound as described earlier.

3.2. General Mean-Variance Pairs For the general case, we propose the use of the bisection search algorithm to find the bound on the expected $k$ th-order statistic by solving $\min _{z} f_{k: n}(z)$. The lower and upper bounds on the range of the optimal $z *$ to initialize the bisection search method in this case reduces to

$$
z_{u}=\max _{1 \leq i \leq n}\left(\mu_{i}+\sigma_{i} \frac{2 k-n-2}{2 \sqrt{(k-1)(n-k+1)}}\right)
$$

and

$$
z_{l}=\min _{1 \leq i \leq n}\left(\mu_{i}+\sigma_{i} \frac{2 k-n-2}{2 \sqrt{(k-1)(n-k+1)}}\right) .
$$

THEOREM 7: Two closed form upper bounds on the expected value of the kth-order statistic given $\boldsymbol{X} \sim_{\theta}\left(\boldsymbol{\mu}, \boldsymbol{\sigma}^{2}\right)$ are

$$
\begin{aligned}
& Z_{k: n}^{*} \leq f_{k: n}\left(\max _{1 \leq i \leq n}\left(\mu_{i}+\sigma_{i} \frac{2 k-n-2}{2 \sqrt{(k-1)(n-k+1)}}\right)\right), \\
& Z_{k: n}^{*} \leq f_{k: n}\left(\min _{1 \leq i \leq n}\left(\mu_{i}+\sigma_{i} \frac{2 k-n-2}{2 \sqrt{(k-1)(n-k+1)}}\right)\right) .
\end{aligned}
$$

\section{COMPUTATIONAL RESULTS}

In this section, we evaluate the quality of the various bounds proposed in this article. The first example is an application of the highest-order statistic bound in a financial context. The second example is a simulation experiment to compare the performance of the bounds for the general $k$ th-order statistic. The computations were conducted on a Pentium II (550 MHz) Windows 2000 platform with the total computational time under a minute.

\subsection{Application in Option Pricing}

One of the central questions in financial economics is to find the price of a derivative security given information on the underlying assets. Under a geometric Brown- 
ian motion assumption on the prices of the underlying assets and using the no-arbitrage assumption, the Black-Scholes [7] formula provides an insightful answer to this question. Assuming no arbitrage, but without making specific distributional assumptions, Lo [16], Bertsimas and Popescu [6], and Boyle and Lin [8] derived moment bounds on the prices of options. Our particular focus is on finding bounds on the price of an option known as the lookback option under moment information on the asset prices.

Let $x_{1}, x_{2}, \ldots, x_{n}$ denote the price of an asset at $n$ different times. A simple lookback European call option on these assets with strike price $K \geq 0$ has a payoff of $\max \left(X_{n: n}-K, 0\right)$. Let $r$ denote the risk-free interest rate and $T$ denote the maturity date. Under the no-arbitrage assumption, the price of the lookback option is

$$
P(K)=e^{-r T} E_{\theta}\left[\max \left(X_{n: n}-K, 0\right)\right] \text {, }
$$

where the expectation is taken over the martingale measure. Clearly, the price of this option depends on the highest-order statistic. Under mean and variance information on $X_{i}$, Boyle and Lin [8] proposed the following upper bound on the price of the lookback option:

$$
P(K) \leq e^{-r T} \sum_{i=1}^{n} \frac{1}{2}\left[\mu_{i}-K+\sqrt{\left(\mu_{i}-K\right)^{2}+\sigma_{i}^{2}}\right] .
$$

We use the results from Section 2 to find the best bounds on $P(K)$. Note that although the asset prices are nonnegative in practice, we do not model this explicitly here to compute our bounds.

The specific lookback option-pricing example is taken from Andreasen [1]. An upper bound on the price of a European call lookback option over $n=10$ time steps is calculated. The risk-free interest rate $(r)$ is $5 \%$ and the time to maturity $(T)$ is 1 year. Table 1 provides the mean and variance information of the asset prices over the 10 periods.

The bounds on the option price are computed for strike prices $K$ from 70 to 140 in steps of 10. Table 2 provides six bounds under mean-variance information and

TAвLE 1. Mean-Variance Data on Asset Prices

\begin{tabular}{lccccc}
\hline Asset Price $X_{i}$ & Mean $\mu_{i}$ & Variance $\sigma_{i}^{2}$ & Asset Price $X_{i}$ & Mean $\mu_{i}$ & Variance $\sigma_{i}^{2}$ \\
\hline 1 & 100.50 & 40.48 & 6 & 103.05 & 257.92 \\
2 & 101.00 & 81.94 & 7 & 103.56 & 304.55 \\
3 & 101.51 & 124.4 & 8 & 104.08 & 352.26 \\
4 & 102.02 & 167.87 & 9 & 104.60 & 401.08 \\
5 & 102.53 & 212.37 & 10 & 105.13 & 451.03 \\
\hline
\end{tabular}

Source: Andreasen [1]. 
TABLE 2. Upper Bound on Lookback Call Option Price

\begin{tabular}{|c|c|c|c|c|c|c|c|c|}
\hline \multirow[b]{2}{*}{ Bound } & \multicolumn{8}{|c|}{$K$} \\
\hline & 70 & 80 & 90 & 100 & 110 & 120 & 130 & 140 \\
\hline Tight mean-variance bound (16) & 75.38 & 65.87 & 56.35 & 46.84 & 37.33 & 27.81 & 19.58 & 14.82 \\
\hline Our closed-form bound (17) & 78.00 & 68.49 & 58.98 & 49.46 & 39.95 & 30.44 & 20.93 & 14.82 \\
\hline Our closed-form bound (18) & 85.49 & 75.97 & 66.46 & 56.95 & 45.71 & 28.14 & 19.58 & 14.82 \\
\hline Boyle and Lin's bound (33) & 327.97 & 238.52 & 154.36 & 84.85 & 45.71 & 28.14 & 19.58 & 14.82 \\
\hline Arnold and Groeneveld's bound (1) & 81.20 & 68.46 & 57.00 & 47.06 & 38.79 & 32.12 & 26.88 & 22.80 \\
\hline Aven's bound (3) & 77.79 & 68.28 & 58.77 & 49.25 & 44.38 & 44.38 & 44.38 & 44.38 \\
\hline Tight mean-variance-covariance bound (22) & 73.23 & 63.73 & 54.25 & 44.79 & 35.40 & 26.41 & 19.30 & 14.75 \\
\hline
\end{tabular}

Source: Andreasen [1]. 
an additional bound under covariance information. For the last bound, we assumed that the asset prices were uncorrelated and solved (22) with the semidefinite optimization code SeDuMi. From Table 2, it is observed that Boyle and Lin's bound is very loose for small values of $K$. On average, our proposed closed-form bound (17) outperforms both Arnold and Groeneveld's [3] and Aven's [4] bound, respectively. Although the closed-form bound (18) is weaker for smaller $K$, it is in fact tight for larger $K$, indicating its usefulness. In Figure 1, we provide the graphical comparison of the bounds (excluding Boyle and Lin's [8] bound, which is tight only for large $K)$.

\subsection{Simulation Test}

The second example is a simulation test to compare the relative performance of the different bounds under randomly generated moment information. We consider $n=30$ random variables. The mean-variance pairs for each random variable were independently chosen from a uniform distribution with $\mu_{i} \sim U[0,50]$ and $\sigma_{i}^{2} \sim$ $U[100,400]$. One hundred mean-variance pairs were sampled in these ranges and

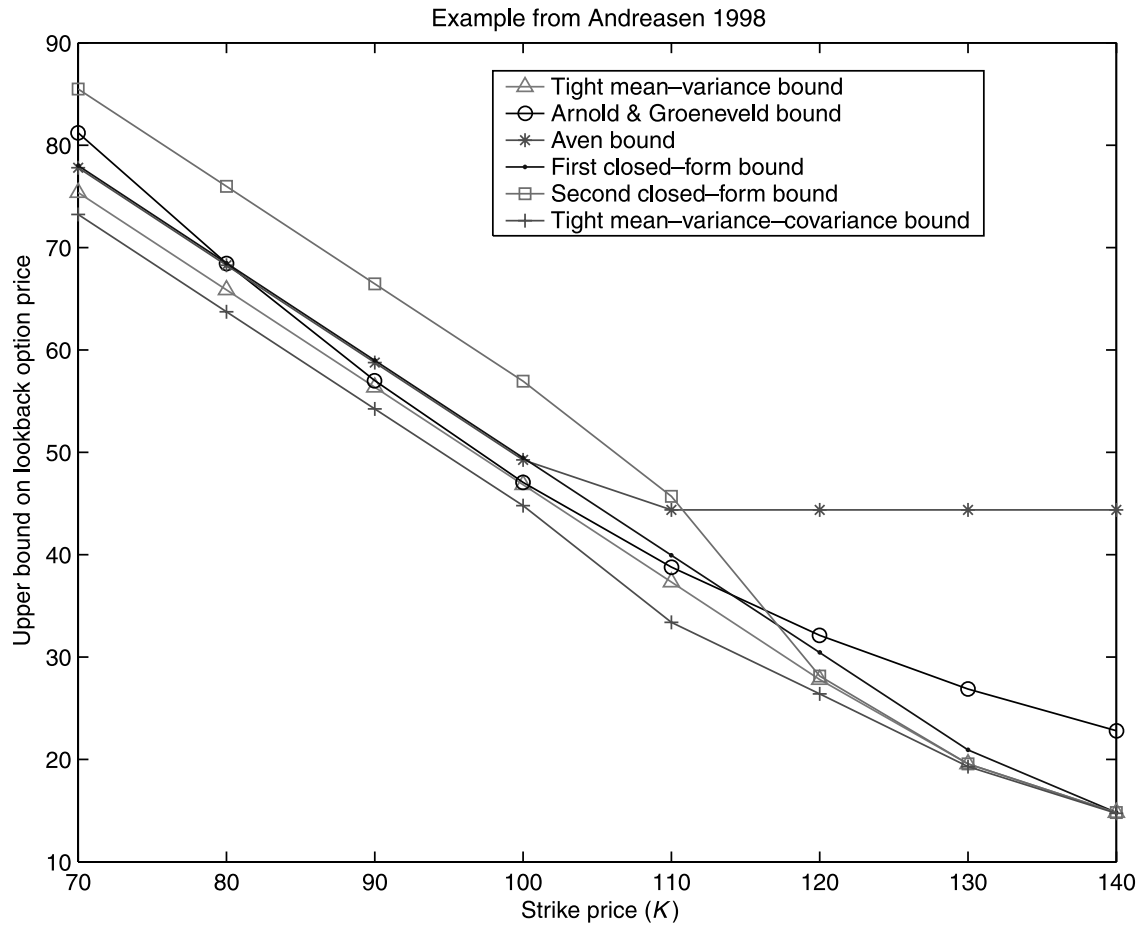

FigURE 1. Upper bound on lookback call option price. From Andreasen [1]. 
the bounds on the expected order statistics were computed. For each closed-form bound, we evaluate the relative percentage error:

$$
\text { Percentage error }=\left(\frac{\text { Closed form bound }- \text { Bisection search bound }}{\text { Bisection search bound }}\right) \times 100 \% \text {. }
$$

For the highest-order statistic, the percentage error of the bounds are provided in Figure 2 and Table 3.

Note that in this case, the bisection search method finds the tight bound $Z_{n: n}^{*}$. In this case, our closed-form bound (13) performs the best and bound (14) is relatively weaker.

We next consider the results for a lower-order statistic. Since the upper bound for the lowest-order statistic $Z_{1: n}^{*}$ from (23) simply reduces to $\sum_{i=1}^{n} \mu_{i} / n$, we use the second lowest-order statistic $Z_{2: n}^{*}$ to compare the bounds. For this case, the bisection search method does not guarantee finding the tight bound. The results obtained are presented in Figure 3 and Table 4. For this case, our closed-form bound (14) is

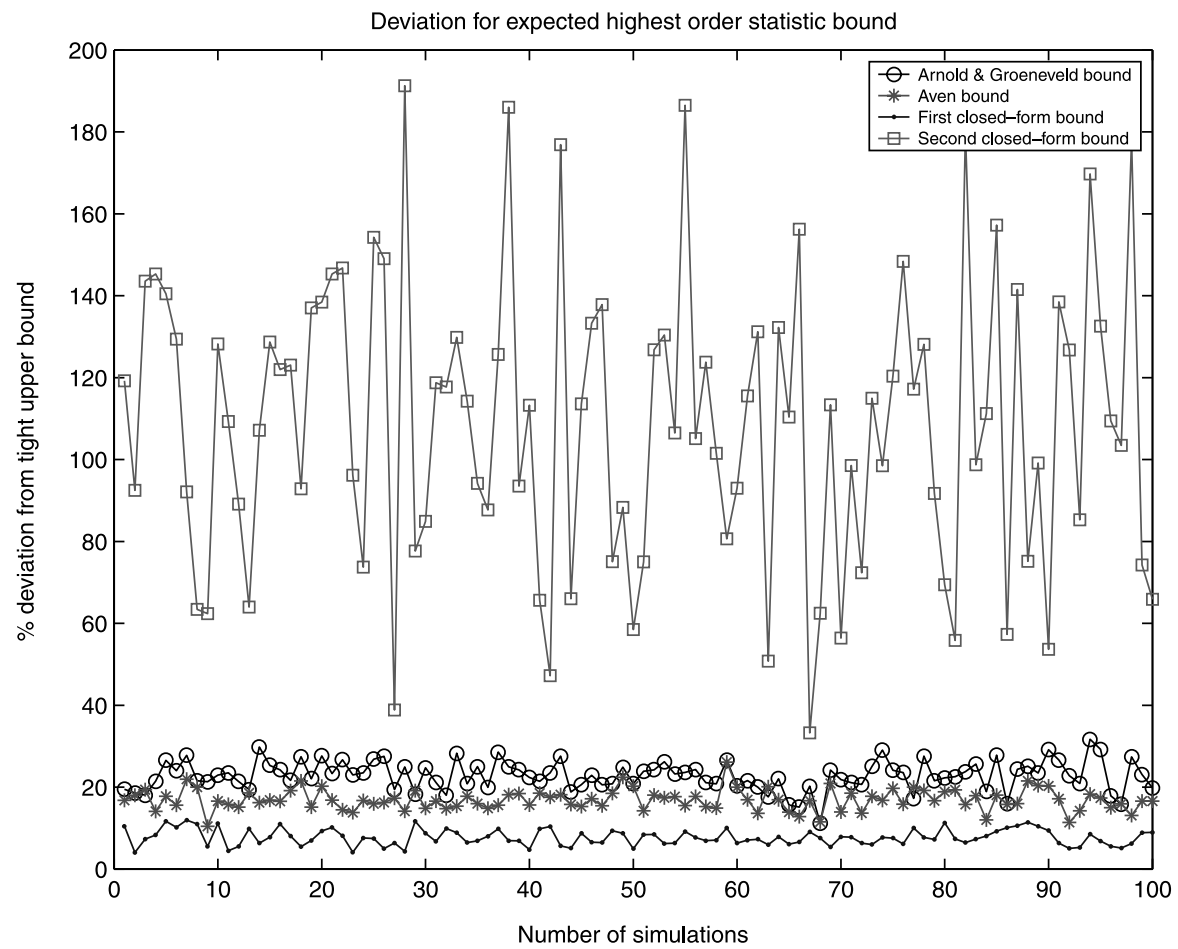

Figure 2. Deviation of closed-form bounds from tight bound on expected highestorder statistic. 
TABLE 3. Statistics of Deviation of Closed-Form Bounds for Expected Highest-Order Statistic

Bound

Mean \% Error

Std. Dev. \% Error

Our closed-form bound (13)

Our closed-form bound (14)

7.73

108.93

35.57

Arnold and Groeneveld's bound (1)

22.86

3.64

Aven's bound (3)

16.91

2.56

observed to be tightest among the closed-form bounds, with an average percentage error of about $1 \%$.

The simulation results seem to indicate that the two closed-form bounds perform well in reasonable settings. Interestingly, in each of the two simulations, the best-closed form bounds were observed to be one of our bounds. Although

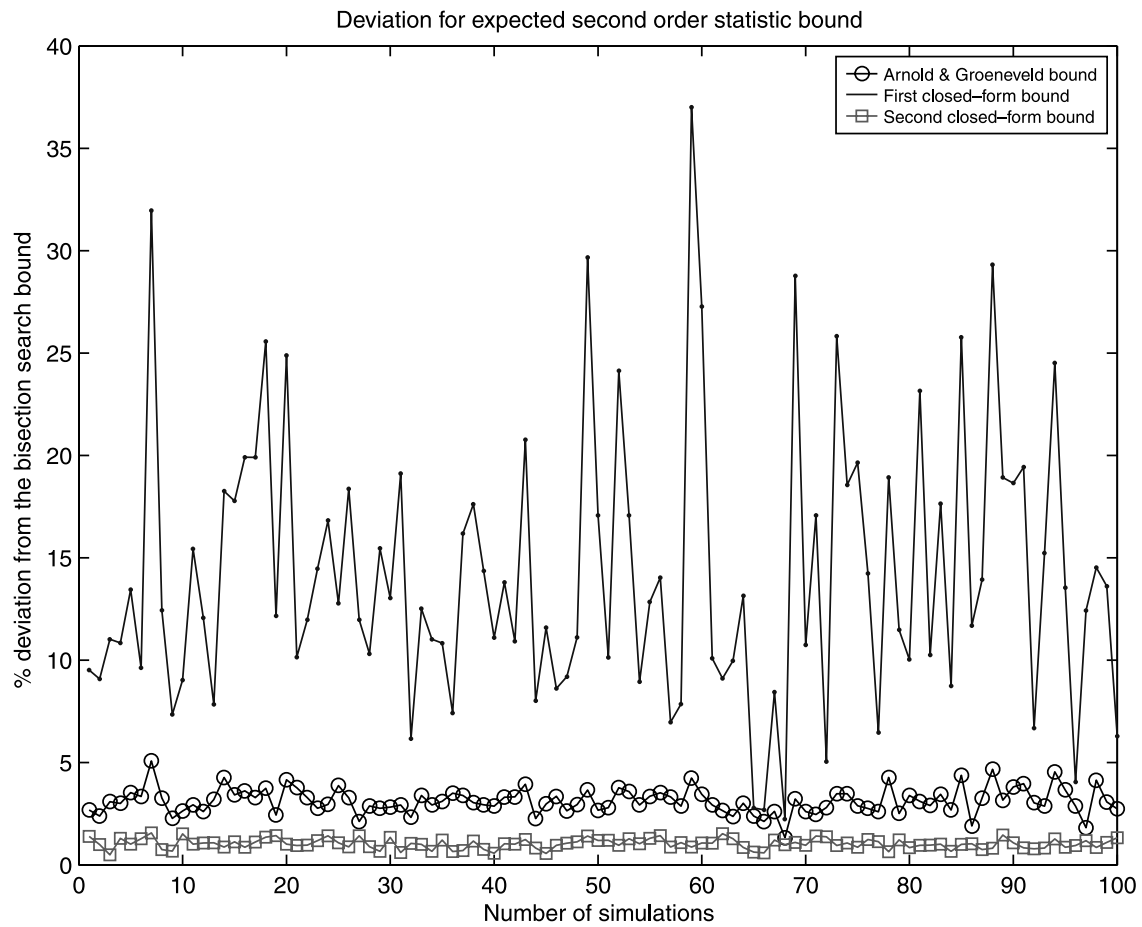

FigUre 3. Deviation of closed-form bounds from bisection bound on secondorder statistic. 
TABLE 4. Statistics of Deviation of Closed-Form Bounds for Expected Second-Order Statistic

\begin{tabular}{lcc}
\hline Bound & Mean \% Error & Std. Dev. \% Error \\
\hline Our closed-form bound (30) & 14.21 & 6.74 \\
Our closed-form bound (31) & 1.04 & 0.25 \\
Arnold and Groeneveld's bound (1) & 3.13 & 0.63
\end{tabular}

cases can be constructed for which both of the bounds are weaker than either Arnold and Groeneveld's and Aven's bounds, the results suggest that the bounds are useful.

\section{SUMMARY}

In this article, we studied the problem of finding tight bounds on the expected value of order statistics under first and second moment information on the random variables. For the highest-order statistic, we showed that the tight upper bound could be found efficiently under mean-variance information with a bisection search method and under mean-variance-covariance information with semidefinite programming. For the general $k$ th-order statistic, we provided efficiently computable bounds (not necessarily tight) under mean-variance information. Finding tight bounds for the general $k$ th-order statistic under mean-variance and possibly covariance information is a potential research area for the future.

\section{Acknowledgment}

This research was supported in part by the Singapore-MIT alliance.

\section{References}

1. Andreasen, J. (1998). The pricing of discretely sampled Asian and lookback options: A change of numeraire approach. Journal of Computational Finance 2(1): 5-30.

2. Arnold, B.C. \& Balakrishnan, N. (1989). Relations, bounds and approximations for order statistics. Lecture Notes in Statistics No. 53. Berlin: Springer-Verlag.

3. Arnold, B.C. \& Groeneveld, R.A. (1979). Bounds on expectations of linear systematic statistics based on dependent samples. Mathematics of Operations Research 4(4): 441-447.

4. Aven, T. (1985). Upper (lower) bounds on the mean of the maximum (minimum) of a number of random variables. Journal of Applied Probability 22: 723-728.

5. Bertsimas, D., Natarajan, K., \& Teo, C.-P. (2004). Probabilistic combinatorial optimization: Moments, semidefinite programming and asymptotic bounds. SIAM Journal of Optimization 15(1): 185-209.

6. Bertsimas, D. \& Popescu, I. (2002). On the relation between option and stock prices: A convex optimization approach. Operations Research 50(2): 358-374.

7. Black, F. \& Scholes, M. (1973). The pricing of options and corporate liabilities. Journal of Political Economy 81: 637-654.

8. Boyle, P. \& Lin, X.S. (1997). Bounds on contingent claims based on several assets. Journal of Financial Economics 46: 383-400. 
9. David, H.A. \& Nagaraja, H.N. (2003). Order statistics, 3rd ed. New York: Wiley.

10. Gumbel, E.J. (1954). The maximum of the mean largest value and of the range. Annals of Mathematical Statistics 25: 76-84.

11. Hartley, H.O. \& David, H.A. (1954). Universal bounds for mean range and extreme observations. Annals of Mathematical Statistics 25: 85-89.

12. Isii, K. (1963). On the sharpness of Chebyshev-type inequalities. Annals of the Institute of Statistical Mathematics 14: 185-197.

13. Jagannathan, R. (1976). Minimax procedure for a class of linear programs under uncertainty. Operations Research 25(1): 173-176.

14. Karlin, S. \& Studden, W.J. (1966). Tchebycheff systems: With applications in analysis and statistics. New York: Wiley-Interscience.

15. Lai, T.L. \& Robbins, H. (1976). Maximally dependent random variables. Proceedings of the National Academy of the Sciences of the United States of America 73(2): 286-288.

16. Lo, A.W. (1987). Semi-parametric upper bounds for option prices and expected payoffs. Journal of Financial Economics 19: 373-387.

17. Meilijson, I. \& Nadas, A. (1979). Convex majorization with an application to the length of critical path. Journal of Applied Probability 16: 671-677.

18. Moriguti, S. (1951). Extremal properties of extreme value distributions. Annals of Mathematical Statistics 22: 523-536.

19. Nesterov, Y. \& Nemirovkii, A. (1994). Interior point polynomial algorithms for convex programming. Studies in Applied Mathematics 13. Philadelphia: Society for Industrial and Applied Mathematics.

20. Parillo, P.A. (2000). Structured semidefinite programs and semi-algebraic geometry methods in robustness and optimization. PhD thesis, California Institute of Technology.

21. Ross, S.M. (2003). Introduction to probability models, 8th ed. New York: Academic Press.

22. Scarf, H. (1958). A min-max solution of an inventory problem. In K.J. Arrow, S. Karlin, \& H. Scarf (eds.). Studies in the mathematical theory of inventory and production. Stanford, CA: Stanford University Press, pp. 201-209.

23. Sturm, J.F. SeDuMi version 1.03. Available from http://sedumi.mcmaster.ca/. 